\title{
Die Relevanz der deutschen Internationalen Beziehungen und die Notwendigkeit polit-ökonomischer Konfliktanalyse
}

\author{
Kai Koddenbrock, Universität Bayreuth
}

Im Erscheinen in:

Bergem W. und H. Schöne (Hg) (2021). Wie relevant ist die Politikwissenschaft? Berlin: Springer

Die Disziplin der Internationalen Beziehungen in Deutschland hat wie die Politikwissenschaft in ihrer Breite eine Fokussierung auf Theorien und Analysen mittlerer Reichweite durchgemacht. Dieser Konsens folgte auf Jahrzehnte der Selbstvergewisserung in der Nachkriegszeit und der hochpolitisierten 1970er Jahre. Angesichts der offensichtlich konfliktiven Weltmachtkonkurrenz und globaler Abhängigkeitsverhältnisse gewinnen makrotheoretische Perspektiven an Relevanz, die polit-ökonomisch das Zusammenspiel innergesellschaftlicher und weltgesellschaftlicher Prozesse $\mathrm{zu}$ fassen versuchen. Anhand zweier Vignetten zur deutschen Außen- und Außenwirtschaftspolitik und zur Geschichte der Beziehungen zwischen Europa und Senegal illustriert der Aufsatz, wie eine solche politökonomische Analyse für die IB fruchtbar gemacht werden kann.

Geoökonomie, Wirtschaftspolitik, Außenpolitik, Konflikttheorie, Geschichte der Politikwissenschaft

\section{Einleitung ${ }^{1}$}

Die Disziplin der Internationalen Beziehungen, Studentin des „largest and most complicated social system possible“ (Lake 2011, S. 467), steht vor einer besonders schwierigen analytischen Aufgabe. Traditionell hat sie sich auf die zwischenstaatlichen Beziehungen konzeund Golntriert, in den letzten Jahrzehnten in Deutschland vor allem auf internationale Institutionen, Organisationen und Normen. Die Strategie, statt größerer Zusammenhänge Theorien mittlerer Reichweite von Teilphänomenen zu entwickeln, war fachpublikationsstrategisch rational, aber hat den Internationalen Beziehungen (IB) wenig gesellschaftliche Relevanz verschafft. Im Gegenteil.

Auch die Friedens- und Konfliktforschung als wichtiger Teil der Internationalen Beziehungen hat in den letzten Jahrzehnten eine dezidierte Wendung hin zur Forschung über das Konfliktmanagement vorgenommen (Daase 1996; Bonacker 2011), die aus der Logik der 1990er Jahre heraus rational war, als UN-Friedenmissionen und ,humanitäre Interventionen“ die politische Praxis und Analyse dominierten. Heute nehmen angesichts massiv wachsender sozialer Ungleichheit im Zuge häufiger Wirtschaftskrisen sowohl die innergesellschaftliche Polarisierung als auch deren Übersetzung in konfrontatives außenpolitisches Handeln von USA und China bis Griechenland und Türkei zu. Der Bedarf nach Konfliktursachenforschung und

\footnotetext{
${ }^{1}$ Der Verfasser dankt Andreas Nölke, Sebastian Schindler, Sebastian Kohl, Sanja Böhler-Koddenbrock sowie Wolfgang Bergem für hilfreiche Kommentare zu diesem Beitrag. Dank auch an Felix Birkner die Forschungsunterstützung und an Daniel Lambach für Organisation und Einladung zur Tagung zur Relevanz der Politikwissenschaft in Frankfurt am Main im Dezember 2019, für die eine erste Version dieses Textes verfasst wurde.
} 
die nuancierte Analyse der Konflikte selbst, um sie besser verstehen zu können, ist also offensichtlich.

Angesichts des Aufstiegs Chinas, tief integrierter globaler Finanzmärkte, langer Wertschöpfungsketten und der Instabilität der transatlantischen Allianz sind Analyseperspektiven zunehmend gefragt, die die sichtlich konfliktive Weltpolitik und ihre gesellschaftlich und globalen Bedingungen direkt fokussieren. Die neue Realität der Konflikte wird in deutschen und US-amerikanischen Think Tanks wie der Deutschen Gesellschaft für Auswärtige Politik (DGAP) oder dem Council on Foreign Relations bereits reflektiert (Schwarzer 2020). Dies ist vor allem eine Reaktion auf den spürbaren Wandel der USAußenpolitik, die durch geoökonomische Strategien ,War by Other Means` (Blackwill und Harris 2016) betreibt und Vorreiter neuer, aggressiverer Außenpolitik ist.

Eine Rückkehr ,realistischer' Politikanalyse im Sinne E. H. Carrs zeichnet sich ab (vgl. Koddenbrock 2017). Auch Finanzanalysten wie Wolfgang Münchau (2020) sehen deutlich die neuen strategischen Herausforderungen deutscher Außen(wirtschafts)politik zwischen USA, Russland und China. Aber die disziplinären Debatten in deutscher IB- und Konfliktforschung verharren weitgehend in den Kämpfen der 1990er und 2000er Jahre, als sich die wichtigsten Fragen um die UN, um internationale Normen wie die ,responsibility to protect' oder Wohl und Wehe des UN-Peacekeepings drehten.

Ein konfliktanalytischer Zugang, der dem Gegenstand der Internationalen Beziehungen gerecht wird, müsste also die Weltordnung als ,größtmögliches und kompliziert möglichstes Sozialsystem “ (Lake 2011, Übersetzung KK) in ihren konstitutiven und dynamischen Komponenten zu verstehen suchen. Gleichzeitig müsste dieser Zugang sich dafür interessieren, wie sich dieses ,System“ aus innergesellschaftlichen und außergesellschaftlichen, zwischenstaatlichen und international-institutionellen Prozessen und Konflikten ergibt. Diese Art der großen und synthetisierende Konfliktanalyse, die trotzdem innergesellschaftliche Prozesse mitreflektiert, findet sich zum Beispiel in den Arbeiten des Wirtschaftshistorikers Adam Tooze und der im deutschen Kontext trotz seiner einzigartigen Art und Weise, Sicherheit und Wirtschaft zusammenzudenken, völlig vernachlässigte Gilbert Ziebura. ${ }^{2}$

Adam Tooze's regelmäßige Analysen der sicherheits- und wirtschaftspolitischen Großwetterlage in Foreign Policy und der London Review of Books basieren auf einer Synthese von sicherheits- und wirtschaftspolitischem Denken, die in Deutschland aktuell nicht zu finden ist. In Is this the end of the American century: America Pivots (2019) und Whose century: After the Shock (2020) widmet sich Tooze der klassischen Frage nach der amerikanischen Dominanz, die IB- und Konfliktforscher*innen schon seit Ende des Zweiten Weltkrieges beschäftigt hat. Seine Analyse zeichnet aus, dass er immer die militärischen und wirtschaftlichen Pfeiler amerikanischer, chinesischer und europäischer Macht zusammendenkt, ein Ansatz, der mit Gilbert Zieburas Weltwirtschaft und Weltpolitik (1984) in Deutschland seinen letzten Auftritt im universitären System hatte.

Tooze argumentiert, dass die finanziellen und militärischen Pfeiler amerikanischer Macht auch unter Trump noch intakt seien. Der Stellenwert des Dollars und des US-dominierten globalen Finanzsystems sei ungebrochen und der militärisch-strategische Vorsprung kaum geschwunden

\footnotetext{
${ }^{2}$ Die emeritierte polit-ökonomisch geschulte Generation von Ekkehart Krippendorf, Joachim Hirsch und Elmar Altvater bis Hartmut Elsenhans hat auf ihre Weise auch mit diesen Fragen gearbeitet, ihre Arbeiten werden im deutschen universitären IB-Betrieb jedoch ebenfalls kaum mehr rezipiert.
} 
(Tooze 2019). Doch Trumps Berater machten sich auf den Weg, Chinas Herausforderung aktiv zu begegnen. Sie seien überzeugt, dass "while China rose, America slept" (Tooze 2020, S. 8) ,geoeconomics', geopolitics (Babic 2021) und, weaponized interdependence' (Farell und Newman 2019) sind wieder auf der Agenda. Die internationale Ordnung wird in den Augen dieser Strateg*innen nicht mehr primär von internationalen Institutionen stabilisiert, sondern aktive realistische Außen- und Außenwirtschaftspolitik im Wettbewerb der Mächte rücken ins Zentrum.

Gilbert Ziebura hat in Weltwirtschaft und Weltpolitik:1922/24-1931 im Jahre 1984 ein in der deutschen IB- und Konfliktforschung fast völlig unbeachtetes Meisterwerk verfasst, das auch Tooze zu Papier gebracht haben könnte. Ziebura kombiniert in seiner Analyse der USamerikanischen und europäischen Politik makroökonomische Strukturanalyse mit minutiösem Blick für politische Entscheidungspozesse und Akteurshandeln. Ähnlich wie Tooze heute analysiert er die USA als ,Scharnier-Macht“ (Ziebura 1984, S. 32), die die politischen und ökonomischen Machtzentren USA-Asien und USA-Europa im Eigeninteresse weitestgehend mitzusteuern versucht.

Mit Ziebura gewinnen die Pfeiler polit-ökonomischer IB Kontur: Politische Entscheidungen und weltwirtschaftliche Zusammenhänge sind Teil grundlegend kapitalistischer Reproduktion, werden aber nicht von dieser überdeterminiert. Aber die kapitalistischen Strukturlogiken müssen mitreflektiert werden. Diese sind nun grundsätzlich krisenhaft und bringen notwendigerweise Verteilungskämpfe mit sich. Diese polit-ökonomische Sensibilität für Verteilungskämpfe um Macht und Wohlstand ist nötig, um den Zusammenhang zwischen innergesellschaftlichen und globalen Prozessen überhaupt erst analytisch fassen zu können. So hat außen- und außenwirtschaftspolitisches Verhalten unterschiedliche Effekte auf Binnenoder exportorientierte Sektoren der eigenen Wirtschaft und damit direkte Wohlfahrtseffekte. Gleichzeitig ist davon auszugehen, dass die Artikulation der betreffenden Interessen zu politischen Konflikten führt, die politisch befriedet werden müssen. Durch die Verbindung von mikro- und makroskopischer Sensibilität für die grundsätzliche Konflikthaftigkeit (welt)gesellschaftlicher Beziehungen werden politische Konflikte und ihre Verbindung zu Verteilungsfragen erst verstehbar.

Betrachten wir die IB pluralistisch als inhärent in Symbiose mit der Friedens- und Konfliktforschung und der Internationalen Politischen Ökonomie (Brühl 2012; Schlichte 2012), finden Fragen nach globalen Konflikten um Wohlstand und Status ganz selbstverständlich einen Platz. Aus Sicht von Internationalen Beziehungen und Konfliktforschung braucht es also eine Analyse des Regierungshandelns und seiner gesellschaftlichen und globalen Bestimmungsfaktoren (ten Brink 2008; Koddenbrock 2018).

In diesem Beitrag werde ich nun einerseits untersuchen, ob diese polit-ökonomische Perspektive auf internationale Beziehungen und internationale Konflikte an der Schnittstelle von ,structure‘ und ,agency“ (Wendt 1987) im deutschsprachigen Raum tatsächlich abwesend ist. Nachdem ich diese Frage im nächsten Abschnitt durch eine kurze Geschichte der deutschen Politikwissenschaft und der Internationalen Beziehungen bejaht habe, werde ich anhand zweier kurzer Vignetten illustrieren, was eine sowohl mikro- als auch makrosensible, politökonomische Konfliktanalyse internationaler Politik zutage fördern kann.

Die erste Vignette widmet sich der angesichts der Vergiftung des russischen Oppositionellen Nawalny im August 2020 intensivierten Debatte um die Bestimmungsfaktoren deutscher 
Außenpolitik. Ich werden zeigen, dass außenwirtschaftspolitische Erwägungen eindeutig überwiegen, aufgrund der theoretischen und thematischen Schwerpunktsetzungen der IB aber selten erkannt werden. Die zweite Vignette illustriert die polit-ökonomische Konfliktanalyse anhand der Geschichte der Handelsbeziehungen des Senegals. Das Zusammenspiel wirtschaftlicher Aktivitäten, kolonialer Administration und militärischer Gewalt trug dazu bei, Gesellschaft und Wirtschaft des Senegals so nachhaltig zu transformieren, dass es der senegalesischen Regierung erst in den letzten 20 Jahren gelingt, ihren Handlungsspielraum langsam auszubauen, der aber weiterhin durch die Abhängigkeit von ausländischen Finanzmitteln und Exporterlösen prekär bleibt.

\section{Zur Geschichte von Politikwissenschaft und Internationalen Beziehungen in Deutschland}

Der Blick in die Geschichte von Politikwissenschaft und Internationalen Beziehungen zeigt ihr Geburtsdilemma: Sie sind bei vermeintlicher Werturteilsfreiheit entweder zu nahe an der bestehenden Politik oder stehen ihr zu kritisch, gar revolutionär gegenüber, um noch als Staatsbetrieb von ihr finanziert zu werden. Abhängig von den spezifischen gesellschaftlichen Bedingungen in den Zwischenkriegsjahren, über die Orientierung suchende Zeit nach Ende des Naziregimes, die linken 1968er, die neoliberalen Jahre nach 1989 und die Jahre von Finanzund Klimakrise seit 2007 galten jeweils unterschiedliche Formen der Politikwissenschaft als angemessen und/oder gefährlich.

Die Politikwissenschaft in Deutschland hat Phasen höchster Politisierung durchlebt, in der, die Gesellschaft` doppelt wichtig war. Zunächst nach 1945 als ,Erziehungsgegenstand“, dann ab den 1970er Jahren als Objekt der Kritik. Als Demokratiewissenschaft nach der Nazizeit neugegründet, hatte die Politikwissenschaft zunächst eine dezidiert normativ-politische Aufgabe gegenüber der Gesellschaft (Bleek 2001). In den 1960er/70er Jahren der Bildungsexpansion und Studierendenbewegung wurde dann explizit über konkurrierende Wissenschaftsverständnisse diskutiert. Ein Verständnis von Wissenschaft als Kritik der Gesellschaft und der ihr inhärenten Herrschaftsformen bildete sich heraus.

Institutionell hat die Politikwissenschaft viele Jahrzehnte des Wachstums und der Konsolidierung als legitime wissenschaftliche Disziplin hinter sich. Die Wende 1989/90 brachte 50 weitere Lehrstühle hinzu und hat der Politikwissenschaft eine dauerhafte Repräsentation in der Breite verschafft. Das aktuelle Selbstverständnis als Disziplin, von dieser Konsolidierung und Normalität geprägt, ist jedoch erstaunlich schwer zu rekonstruieren. Es gibt heute zahlreiche Einführungen in die Teildisziplinen der Politikwissenschaft, sei dies nun die Vergleichende Politikwissenschaft, die Internationalen Beziehungen oder die Politische Theorie, aber kaum mehr den Versuch der Gesamtschau. Viele Politikwissenschaftler*innen scheinen sich weniger als Vertreter*innen des Faches in der Breite denn als Spezialist*innen zu verstehen.

Wilhelm Bleek sah schon 2001 in der letzten großen Gesamtschau Geschichte der Politikwissenschaft in Deutschland die Entwicklung in Richtung von Teilpolitologien skeptisch. „,Diese Entwicklung von Teilpolitologien fördert sicherlich deren empirische und theoretische Kompetenz, führt aber auf der anderen Seite zu Spezialgebieten, die auf Grund ihrer Fragmentierungen und Abschottungen für die Entwicklung der Gesamtdisziplin nicht 
unproblematisch sind“" (Bleek 2001, S. 335). Eckhard Jesses und Sebastian Liebolds Deutsche Politikwissenschaftler - Werk und Wirkung (2014) verschafft ein paar Einblicke neueren Datums.

Von den 1970ern, über die 1980er bis in die 1990er Jahre war die Beschäftigung mit dem Fach intensiver. Klaus von Beyme organisierte 1986 ein PVS-Sonderheft zum Thema Politikwissenschaft in der Bundesrepublik Deutschland. Entwicklungsprobleme einer Disziplin. Gerhard Göhler und Bodo Zeuner veröffentlichten 1991 Kontinuitäten und Brüche in der deutschen Politikwissenschaft und in den 1970er Jahren, die von den hoch politisierten Kämpfen zwischen linken und eher konservativen Ordinarien bestimmt waren, war Die Entwicklung der westdeutschen Politikwissenschaft, 1977 von PROKLA-Redakteur Hans Kastendiek veröffentlicht, keineswegs die einzige Publikation der Selbstvergewisserung.

Man kann diese Werke als Indiz dafür verstehen, dass Ausrichtung und Charakter der Politikwissenschaft umstritten waren. Diese Bücher wurden geschrieben, um diese Ausrichtung zu klären und zu beeinflussen. Dass sie nicht mehr geschrieben werden, erscheint als Anzeichen für mangelnde Umstrittenheit und fehlenden Streit. Positiv gewendet indiziert dies die professionelle Etabliertheit politikwissenschaftlicher Teildisziplinen, die sich durch spezialisierte Auseinandersetzung an kleineren Gegenständen entwickeln. Große, relevante politische Grundsatzfragen kommen dabei nur noch selten in den Blick. Was in dieser Ruhe ebenfalls vergessen wird, ist die notwendige Umstrittenheit von Politik und Politikwissenschaft. Auf Politik gibt es keine ,view from nowhere'. Der mangelnde Streit ist also ein Krisenphänomen, da wissenschaftliches Feld und reale Politik an gegenseitiger Passung verloren haben. In der Politik wird sichtlich gerungen, in der Politikwissenschaft, insbesondere in den IB jedoch nicht.

Der mangelnde Fokus auf große und grundsätzliche Fragen wird auch in den IB als Frage nach großer Theorie allerorten gestellt - ohne bisher in Deutschland große Konsequenzen gehabt zu haben. Das einflussreiche European Journal of International Relations organisierte 2014 ein ganzes Symposium zum Thema. 2016 fragte Dan Drezner in der Washington Post: , Where have all the big international relations theories gone?` Auch die Frankfurter Kolleg*innen Nicole Deitelhoff und Christopher Daase stellten sich auf dem großen ZIB-Roundtable in Bremen 2017 die Frage nach der Relevanz der IB und konstatierten, dass diese ,ihren eigenen Wahrheitsanspruch kleingeredet, die Möglichkeit gesicherten Wissens bezweifelt und ihre Analyse in einem reflexiven Regress auf die eigene Arbeit konzentriert" (2017) habe. Gerade der ,reflexive Regress auf die eigene Arbeit' scheint mir zentral. Die Hinwendung zur realen Politik, zu ihrer realen Konflikthaftigkeit steht an (vgl. Schindler 2020). Siegfried Schieder plädierte in Aus Politik und Zeitgeschichte dafür, die Theorie wieder ernster und wichtig zu nehmen, diagnostizierte jedoch überraschend und im Gegensatz zum unten diskutierten TRIPS survey, dass ,[e]tablierte Theorien durch poststrukturalistische Theorien ergänzt“" werden ,und selbst marxistische Theorien, die seit dem Zerfall des Ostblocks als überholt galten, angesichts wirtschaftlicher Krisen eine Renaissance [erfahren]. Selbst das realistische Paradigma ist wieder populär" (Schieder 2018, S. 36). Während der relative Aufstieg poststrukturalistischer Ansätze real ist, bleiben neomarxistische und realistische Perspektiven jedoch weiter stark unterrepräsentiert. Warum eine polit-ökonomische Konfliktanalyse, die damit einherginge, vielversprechend wäre, werde ich im nächsten Abschnitt illustrieren.

Kaum 10 Jahre zuvor, bevor Brexit, Trump und China das liberale Selbstverständnis zu erschüttern begannen, zogen Nicole Deitelhoff und Klaus-Dieter Wolf 2009 ein eher positives 
Resumé zum Zustand der deutschen IB. Der wichtigste Faktor in dieser Konsolidierungsgeschichte war damals für sie der fast konsensuale Fokus auf ,Governance“ (Deitelhoff und Wolf 2009, S. 459). In der Governance-Forschung sahen beide den zentralen Türöffner $\mathrm{zu}$ neuen Themen jenseits der traditionellen Forschung nach gelingender Kooperation und damit der Förderung des Friedens in der Welt. Sie erblickten hinter dieser Tür ,demokratie- und steuerungstheoretische' Bereiche, die auch Fragen von Macht und Herrschaft unter Bedingungen internationaler Anarchie erlaube (ebd.), um zu konstatieren: „Als Mainstream der IB in Deutschland [hat sich] ein lockeres institutionalistisches Theorieparadigma mit rationalistischen und konstruktivistischen Zügen etabliert [...], getragen von einem hermeneutisch informierten ,weichen Positivismus.“ (Deitelhoff und Wolf 2009, S. 461). Der Institutionalismus ist ein Theorieinstrument mittlerer Reichweite, das in alle möglichen Richtungen vom historischen Institutionalismus bis zum Rational-ChoiceInstitutionalismus ausgestaltet werden kann. Das globale „größtmögliche Sozialsystem“ lässt sich damit jedoch nur höchst partiell untersuchen. ,Big theory' ist das dezidiert nicht und große Fragen lassen sich damit nur bedingt stellen und beantworten.

In der ersten größer angelegten Studie zum Charakter der deutschen IB bestätigte sich das Verdikt von Deitelhoff und Wolf aus dem Jahr 2009. Basierend auf dem amerikanischen Teaching, Research and International Policy Survey (TRIPS) von 2016, belegen Wiebke Wemheuer-Vogelaar und Thomas Risse eindrücklich: Der Fokus der deutschen IB liegt auf der institutionalistisch/konstruktivistischen Analyse von Sicherheitspolitik, Internationalen Organisationen und der EU nebst ein wenig IB-Theorie. Die Internationale Politische Ökonomie kommt auf unter $8 \%$ und vergleichende Außenpolitikanalyse auf unter 4\%. Beide Felder sind für eine auch global interessierte Konfliktanalyse, wie ich zeigen werde, jedoch existentiell (vgl. Abb. 1).

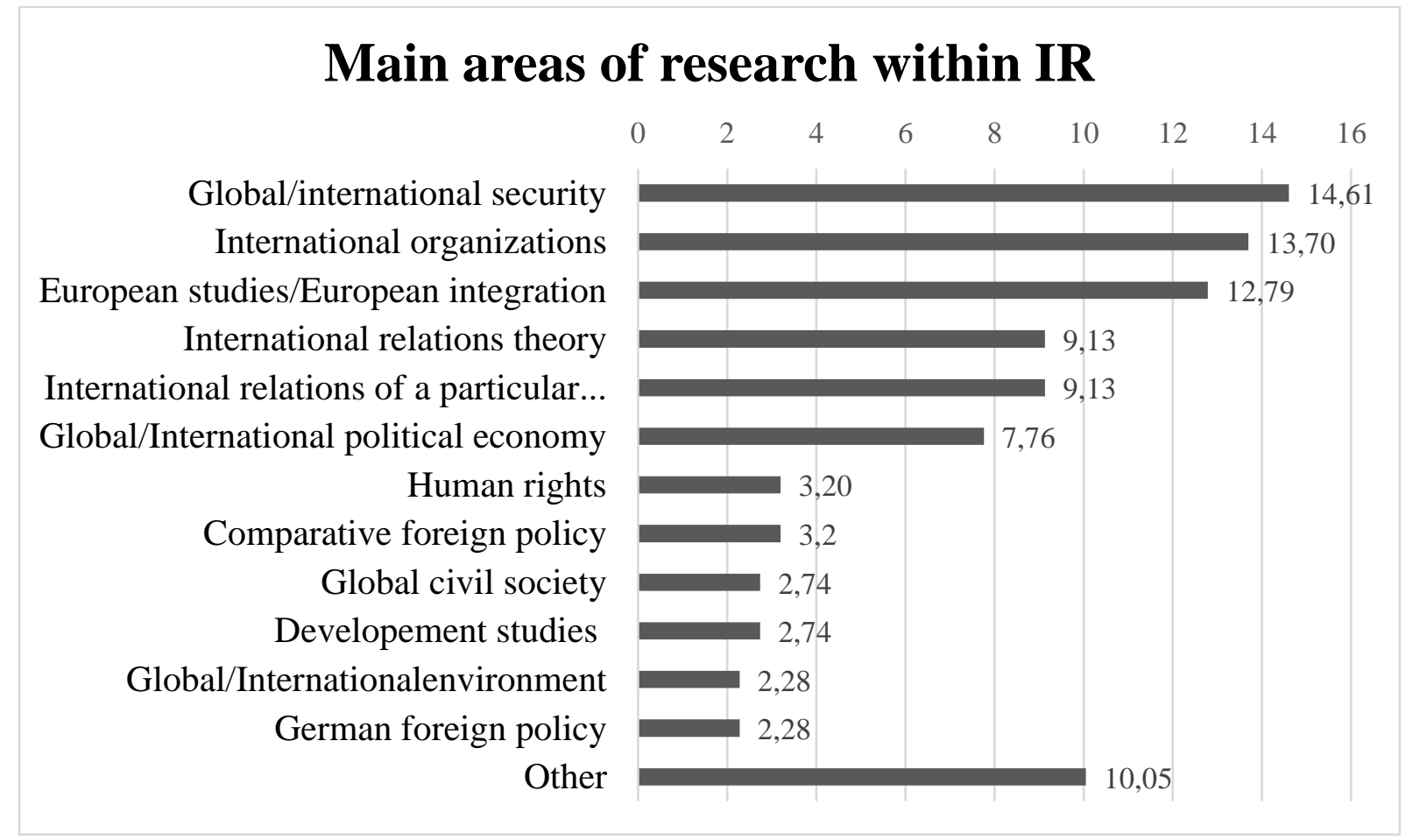

Abbildung 1:Nach Wemheuer-Vogelaar und Risse 2016, S. 95. Zeigt nur Werte über 2\% an. 
,Schulen' und Paradigmen sind ebenfalls eindutig verteilt in der deutschen IB. Die deutschen IB beschäftigt sich mit internationalen Organisationen und der EU aus primär konstruktivistischer Sicht. Außenpolitikanalyse, Marxismus, Feminismus, Entwicklungsländerforschung nehmen nur einen sehr geringen Raum ein (vgl. Abb. 2).

\section{Paradigms/Schools of thought}

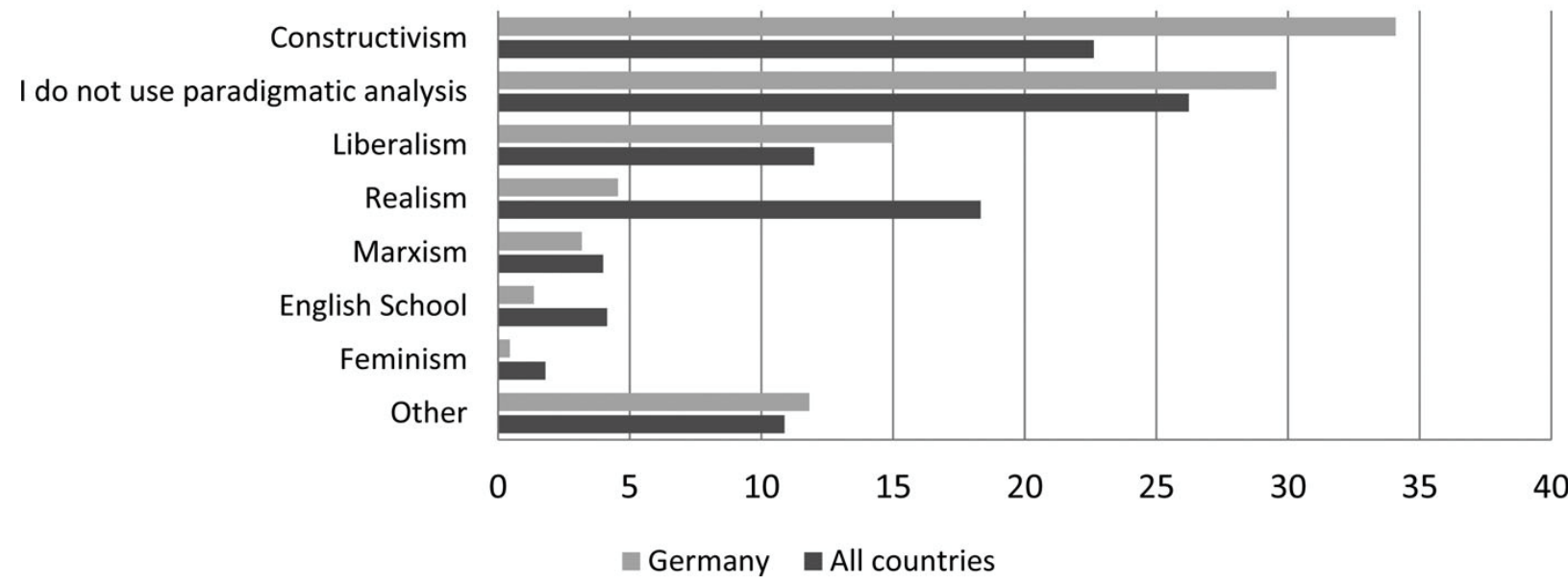

Abbildung 2:Nach Wemheuer-Vogelaar und Risse 2016, S. 95.

Vielfältige Gründe könnten für diese Entwicklung ins Feld geführt werden, aber dies kann hier nicht im Fokus stehen. Feldspezifische Kohorten- und Homogenisierungseffekte angesichts zunehmender Arbeitsplatzunsicherheit und Drittmittelabhängigkeit wären beispielsweise systematisch als Ursache zu untersuchen. Mindestens ebenso wichtig wie mögliche feldspezifische Gründe für den konstruktivistisch-institutionalistischen Zuschnitt der deutschen IB sind aber politische Praxis, gesellschaftliche Mobilisierung und wirtschaftliche Stellung der BRD und die Weise, wie sich diese in Forschungsgegenstände übersetzt.

In Bezug auf die IB beschrieb Wilhelm Bleek zum Beispiel 2001, wie sich parallel zur Etablierung der Entwicklungspolitik die Entwicklungsländerforschung entwickelte. Das habe laut Bleek zu Beginn auch unter dem Vorzeichen, Länder von der Anerkennung der DDR abzuhalten, stattgefunden. In den 1970er Jahren, nach der Studentenbewegung und der Dekolonisierung, gab es dann intensive Theoriedebatten. Seit den 1980er Jahren aber fand eine Hinwendung zu ,speziellen Projekten' statt (Bleek 2001, S. 331). Eine Rückbindung der akademischen Schwerpunkte an politische und gesellschaftliche Entwicklungen besteht.

Systematischer hat dieses Wechselspiel zwischen politischer- und wirtschaftlicher Lage eines Landes und ihrer Übersetzung in Themen der IB Michael Mastanduno durchgespielt. In seinem Aufsatz zu Economy and Security in Statecraft and Scholarship in der Zeitschrift International Organization untersucht er 1998, bisher nie in Deutschland repliziert und auch kaum rezipiert, wie zwischen 1945 und 1995 in der US-amerikanischen Außenpolitik Sicherheit und Wirtschaft zusammen verfolgt wurden und inwiefern sich das in der Disziplin der International Relations niederschlug. Mastanduno argumentiert, dass die Frage nach Integration oder Trennung von polit-ökonomischen, auch auf die Außenwirtschaft fokussierten und sicherheitspolitischen Perspektiven erstens von materiellen und zweitens konstruktivistischen Faktoren abhängt. Die materiellen Faktoren entstammten der jeweiligen internationalen Struktur - bipolar, multipolar, unipolar z.B. und dem tatsächlichen Stand der eigenen Sicherheit und des Wohlstandes. Die 
konstruktivistischen Faktoren beträfen die Wahrnehmung ökonomischer und sicherheitspolitischer Bedrohungen. So würden beide Stränge der Außenbeziehungen immer dann stärker integriert, wenn Bedrohungen und wirtschaftlicher Abstieg plausibel würden. In Phasen von Dominanz und wirtschaftlichem Wohlstand werde es den beiden Feldern erlaubt, sich unabhängig auszutoben, da Synergieverluste zu verkraften seien. Dass US-Regierung und die EU heute angesichts der zunehmend expansiven Politik Chinas und Russlands unter Druck stehen und deshalb eine Integration von politischer und ökonomischer Perspektive auf der Tagesordnung steht, zeichnet sich ab.

\section{Politische Ökonomie als Mikro-Makro-Konfliktforschung}

Die IB in Deutschland hat also heute eine doppelte Blindstelle: Die Außenpolitikanalyse ist außer Mode gekommen (vgl. aber Masala 2016) und wenig repräsentiert. Die Außenwirtschaftspolitik ist darin nochmal marginaler (vgl. aber Dieter 2018). Es ließe sich nun in Anlehnung an Mastanduno folgende These aufstellen: Es gibt in Deutschland keine politökonomische IB à la Ziebura oder Tooze, weil Sicherheits- und Außenwirtschaftspolitik tatsächlich völlig getrennte politische und bürokratische Bereiche sind und eine Kohärenz auch in realpolitischen Schwächesituationen Deutschlands nicht angestrebt wird. Noch mehr: Die deutsche Wirtschaft macht im Ergebnis Außenpolitik und die Regierung unterstützt dies auch siehe zum Beispiel den Wirecard-Fall oder das Festhalten an Nordstream 2 (Goldthau 2016). Die Bedeutung der offiziellen Regierungs-Außenpolitik ist eher symbolisch, so dass das mangelnde Interesse für die Außenpolitik in Deutschland fast schon verständlich wird.

Zwar wird in der marginalen Außenpolitikanalyse eine recht lebhafte Debatte über Deutschland als ,Zivilmacht', die mit ,soft power' ausgestattet ist, geführt (Mello 2019; Masala 2016). Aber ein systematischer Fokus auf die Außenwirtschaftspoliitk fehlt. Das ist analytisch problematisch, denn außenwirtschaftspolitisch wird sichtlich so gehandelt, dass es dem Versuch, Deutschland als wohlwollende soft power darzustellen, zuwiderläuft. Durch seine Außenwirtschaftspolitik bestimmt Deutschland die Geschicke der EU und prägt sein Bild in der Welt, das eigentlich die Aufmerksamkeit der IB erforderte. Aber dafür müsste sie ein Instrumentarium für die Außenwirtschaftspolitikanalyse haben.

\section{1 „Handels- und Wettbewerbsstaat“ Deutschland: Außenpolitik als Außenwirtschaftspolitik}

Deutschland ist heute unbestritten der europäische Hegemon und wird im europäischen Ausland auch so porträtiert. Außenpolitikinteressierte erinnern sich an das Merkel-Bild mit Pickelhaube in der Daily Mail, das einen Tobsuchtsanfall der Bild-Zeitung auslöste. Die Daily Mail schrieb damals: "In theory, the 28 independent nations enjoy equal rights, but in practice it's run by Angela Merkel. There's fancy talk about EU community spirit and a vision of what 'Europe' can be in the world, but the truth is that Merkel is Queen Bee and the 27 other national leaders are her drones." 2014 war auch das Jahr, in dem David Cameron sich für das BrexitReferendum entschied. Merkels Politik konnte von den Brexiteers leicht instrumentalisiert werden. Auch Wolfgang Schäuble war über seine Darstellung als Wehrmachtssoldat in einer Syriza-nahen Tageszeitung Anfang 2015 sicher nicht erfreut. Schäubles EU-Politik war steng genommen Außenwirtschaftspolitik. 
Wir sind es gewohnt, Deutschland vor allem als zurückhaltende außenpolitische „Zivilmacht“ zu sehen, die ihre Friedensziele innerhalb der EU zu erreichen versucht und maximal versucht, ,dabeizusein' (FAZ, 4.2.2020). Helga Haftendorn hat das mal im Zuge der Nachkriegszeit bis zur EU als ,Souveränitätsgewinn durch Souveränitätsverzicht' bezeichnet (Daase und Hellmann 2014, S. 293). Auf den Internet-Seiten des Auswärtigen Amtes bekommt man einen höchst partiellen Einblick in diese deutschen Außenbeziehungen. Folgende „Grundprinzipien“ präsentiert Minister Heiko Maas auf der Website des Außenministeriums: 1) Europäische Integration und Europäische Union; 2) Transatlantische Partnerschaft; 3) Engagement für Frieden und Sicherheit; 4) Demokratie, Rechtsstaatlichkeit und Menschenrechte; 5) Globalisierung und internationale Ordnung (Außenministerium, 2019).

Diese Selbstdarstellung entspricht dem Bild der deutschen Außenpolitik, das in den Leitmedien gern diskutiert wird. Aber machen diese Prinzipien symbolischer Außen- und Sicherheitspolitik die außenpolitischen Beziehungen Deutschlands wirklich verständlich? Prioritär scheint diese Politik für die Regierungshaushälter*innen zumindest länger nicht gewesen zu sein: Hellmann, Wagner und Baumann (2014, S. 242) zeigen in Ihrem Handbuch zur Deutsche(n) Außenpolitik, dass ,sich der Anteil der drei Ministerien mit internationalen Aufgaben von einem gemeinsamen Anteil, der im Jahr 1990 noch bei etwa 21,5 \% lag, auf einen Wert von knapp über $12 \%$ im Jahr 2002 fast halbiert. Im Jahr 2008 lag dieser Anteil bei 14,3\%.

Deutschlands Wahrnehmung und Agieren in der Welt wird erst über sein wirtschaftliches Verhalten verständlich. Deutschland ist ,Handelsstaat' (Rosecrance 1986; Staack 2000), ist ,Wettbewerbsstaat“ (Hirsch 1995). Dass Deutschland als ,Exportweltmeister' so wenig im Hinblick auf seine Außenwirtschaftspolitik analysiert wird, lässt sich nur aus den oben genannten disziplinären Eigendynamiken verstehen. Denn die entscheidenden Beziehungen mit der Außenwelt geht Deutschland über wirtschaftliche Beziehungen ein. Diese werden politisch abgesichert und unterstützt. So sind die Handelsbilanzüberschüsse ein ewiger europäischer und nun sogar transatlantischer Spaltpilz, denn „durch die seit dem Jahr 2000 erwirtschafteten stetig hohen Leistungsbilanzüberschüsse gefährdet Deutschland nicht nur die Zukunft des europäischen Integrationsprozesses, sondern auch die der liberalen Handelsordnung“ (Dieter 2018). Andererseits sind die Import-Export-Beziehungen für Beschäftigung, Wachstum, Profite und am Ende auch die Steuereinnahmen existentiell. Die gesamte gesellschaftliche Reproduktion hängt von einer offenen Wirtschaft wie der deutschen von diesen Beziehungen ab. Die Vergleichende Politische Ökonomie bearbeitet diesen Topos seit Jahrzehnten (Braun 2020).

In Bezug auf Weltmarkt und Außenbeziehungen fallen zahlreiche aktuelle Vorhaben ins Auge, die in der universitären IB kaum zur Sprache kommen. So intensiviert die deutsche Regierung ihre Bemühungen, die deutsche Rohstoffversorgung strategisch abzusichern. In der neuen Rohstoffstrategie des Bundeswirtschaftsministeriums, erst der zweiten nach der Premiere 2010, heißt es, dass die Bundesregierung ,die Möglichkeiten zusätzlicher staatlicher Maßnahmen zur Rohstoffsicherung in einer Auftragsstudie prüfen lasse“ (Bundesregierung 2020). Zunehmend steht auch Afrika auf der Agenda, mindestens zur sogenannten Migrationsbekämpfung. Hier ist ,die Wirtschaft' und die Außenwirtschaftsförderung noch relativ planlos (Erhart und Staack 2019; Banse 2019). Konzeptionen von ,Eurafrika' (Hansen und Jonsson 2014), die die Zukunft von Europa und Afrika als zwingend verschränkt begreifen, könnten bald wieder Konjunktur haben. 
Um die Regierungspolitik gegenüber Russland und China in ihrer Widersprüchlichkeit zu verstehen, aber auch den wachsenden Stellenwert Afrikas begrifflich fassen zu können, benötigt die Konfliktanalyse einen neuen Fokus auf Politische Ökonomie. Afrikas Interaktion mit dem Globalen Norden wiederum ist geprägt von dem mühsamen Abschied von kolonialen Pfadabhängigkeiten, die nun in der zweiten Vignette angedeutet werden.

\subsection{Die Politische Ökonomie der Afrika-Europa-Beziehungen und ihre heutige Relevanz}

Die Verschränkungen zwischen Europa und Afrika haben vom Sklavenhandel über den Kolonialismus bis heute eine Intensität und Wirkung erreicht, die den Kontext jeder Entwicklungspolitik und der Militärmissionen von Mali bis zum Horn von Afrika bilden. Aber IB und Konfliktforschung müssen durch ihren professionalisierten Mid-Range-Fokus meist von diesem strukturellen und historischen Kontext abstrahieren. Das führt im Einklang mit dem oben diskutierten institutionalistisch-konstruktivistischen Fokus zu einer oftmals starken Engführung auf die Aktivitäten von internationalen Organisationen und der von ihnen vertretenen und auf die Reise geschickten Normen. Das fördert relevantes Wissen zu Tage, das aber koloniale Pfadabhängigkeiten kaum einbeziehen kann. In unserer Arbeit zu den Finanzbeziehungen zwischen Europa und Senegal versuchen Ingrid Kvangraven, Ndongo Samba Sylla und ich (2021, unter Begutachtung), diesen Blick zu weiten und die Konflikte zu fokussieren, die die Inwertsetzung der Kolonie damals begleiteten und die Versuche der heutigen senegalesischen Regierung, ihren Handlungsspielraum zu erweitern.

Senegal war seit 1818 französische Kolonie und wurde von einem aus Paris entsendeten Gouverneur regiert. Zunächst waren die Hafenstädte St. Louis und Dakar wichtige Umschlagplätze für den Sklavenhandel. Als dieser um 1848 verboten wurde, begannen die Handelshäuser aus Marseille und Bordeaux, auf Erdnüsse umzusatteln. Zur kreditären Unterstützung wurde 1851 die Banque de Senegal von der Kolonialadministration gegründet, Anteile wurden an die wichtigsten Sklavenhändler, sowohl senegalesische als auch französische, ausgegeben. Diese Bank wurde dann aber bis zum Ende der Kolonialzeit nicht für den Aufbau der senegalesischen Wirtschaft, sondern nur für Kredite gegenüber Exporteuren und Handelskompanien aktiv. Der Handlungsspielraum der senegalesischen Geschäftsleute, die zu Beginn noch gut als Mittelsmänner- und frauen verdient hatten, wurde durch die wachsende Marktmacht der beiden größten Handelshäuser Compagnie Française de L'Afrique Occidental und Société Commerciale de l'Ouest Africain immer weiter minimiert. Öffentliche Investitionen aus Paris waren rar, aber Ende des 19. Jahrhundert gelang es den Handelshäusern, die Kolonialadministration zum Bau eines erweiterten Eisenbahnnetzes zu überreden. Dies und die militärische Expansionspolitik unter Gouverneur Faidherbe (Barrows 1976) führte zu einem massiven Boom in den Erdnussexporten und großen Profiten der Handelshäuser.

Mehrere Mechanismen waren am Werke, um diese für Frankreich einträgliche Konstellation stabil zu halten. Einerseits wirkte die Kolonialadministration insbesondere seit 1920 durch Zwangsarbeit und Kopfsteuern darauf hin, möglichst viele Senegales*innen für die Feldarbeit zu gewinnen (Bernards 2019). Andererseits wurde über Investitionen in den Eisenbahnbau die Logistik verbessert. Das Bankensystem und die Geldversorgung wurde höchst restriktiv von Paris aus gesteuert und Kredite wurden vor allem der Exportwirtschaft vergeben.

Die Unabhängigkeitswelle der späten 1950er Jahre machte auch vor den französischen Kolonien nicht halt, aber es gab große Unterschiede zum Ende des britischen Kolonialreiches. 
Geheime Kooperationsabkommen in wesentlichen Bereichen staatlicher Prärogative schränkten den Handlungsspielraum Senegals außerordentlich ein (Pigeaud und Sylla 2018). Die Militärbasen und die fortdauernde Kolonialwährung Franc CFA waren nur der sichtbarste Ausdruck der nur partiellen gewonnenen Souveränität.

In seinem Klassiker der vergleichenden Politischen Ökonomie Small States in World Markets beschreibt Peter Katzenstein, wie kleinere europäische Staaten wie Österreich, die Schweiz, Norwegen und Schweden mit ihren großen Nachbarn und den Zwängen des Weltmarktes umgehen. Durch kluge Industriepolitik gelang es vielen von ihnen, in mehreren Sektoren wettbewerbsfähig zu werden (Katzenstein 1985). Afrikanische Staaten stehen 35 Jahre später vor ähnlichen Herausforderungen und tun dies auf der Basis von kolonialen Pfadabhängigkeiten, die selten in ihrer Radikalität beschrieben werden (Cramer et al. 2020).

Im Zug der Unabhängigkeit verlor zwar der Export von Erdnüssen an relativem Gewicht, aber Phosphat, Rohöl und Fisch stellten mittelfristig keine wesentlich einträglicheren und auch keine diversifizierten Einnahmequellen dar. Zudem blieb das koloniale Franc CFAWährungsarrangement fast unverändert bestehen und das auf kolonialen Export hin optimierte Bankensystem war schwer zu transformieren. In den Jahren 1960-1980 versuchten die ersten senegalesischen Regierungen dies zwar, aber mit der globalen Wirtschaftskrise in den frühen 1980er Jahren und dem sogenannten ,Volcker-Schock ${ }^{*}$ und den danach einsetzenden Strukturanpassungsprogrammen des IWF waren diese Erfolge wieder verloren. Erst seit den frühen 2000er Jahren ist Senegal in der Lage, auch durch Partnerschaften mit chinesischen Unternehmen seinen Handlungsspielraum wieder etwas auszubauen (Sylla und Koddenbrock 2019).

Heute kämpft die senegalesische Regierung mit einer Weltwirtschaft, auf die sie reagieren muss, und einer heimischen politischen Ökonomie, die sie nur langsam umgestalten kann. Große Investitionen wie der Bau des neuen Flughafens werden mithilfe von Krediten der internationalen Finanzinstitutionen finanziert, von türkischen Firmen durchgeführt und treffen auf Proteste der Bürger*innen, die z. B. den Flughafen wieder nur als für die Export/Importindustrie gebaut ansehen. Interessiert sich die IB für den Globalen Süden und die Konflikte, die Regierungen ausfechten müssen, um sich aus kolonialen Pfadabhängigkeiten zu befreien, müsste sie diese auf historischer Basis zu rekonstruieren beginnen.

\section{Schluss}

Konfliktbearbeitung ist Intervention in soziale Beziehungen, die im Gesellschaftlichen und Zwischenstaatlichen immer auch von Konflikten um Macht und Wohlstand durchzogen sind. Konflikte sind zentraler Bestandteil aktueller Arbeiten in den deutschen IB von Konflikten um Normen (Wiener 2018) bis zu Praktiken (Schindler und Wille 2019). Aber im institutionalistisch-konstruktivistisch dominierten wissenschaftlichen Feld fehlt sowohl die breite Rezeption der realistischen, staatszentrierten Konfliktanalyse (Masala 2016) und, wie ich hier zu illustrieren versucht habe, die Perspektive der Politischen Ökonomie. Mit ihrem Fokus auf Macht und Verteilungsbeziehungen wird das Handeln von Akteuren innerhalb von bestehenden und auch veränderbaren Strukturen verständlich. Das gibt der IB gleichzeitig einen direkteren Zugang zu aktuellen, brennenden politischen Fragen von den Veränderungen in der Weltordnung über die Rolle des Dollars und der Verschuldung im Globalen Süden bis zur Bedeutung der Austeritätspolitik für außenpolitisches Verhalten. 
Relevanz ist eine soziale Konstruktion. In diesem Beitrag habe ich die Relevanzkonstruktion im Feld der deutschen IB der letzten Jahre nachgezeichnet und argumentiert, dass für die Analyse größerer Zusammenhänge und aktueller politische Konflikte im ,größtmöglichen Sozialsystem' polit-ökonomische und auch realistische Ressourcen erforderlich sind. Die Politische Ökonomie als Analyseperspektive, die wirtschaftliche und politische, nationale und globale Prozesse in den Blick nimmt, ist besonders geeignet, deutsches außenpolitisches Handeln oder die Transformation von kolonialen Gesellschaften $\mathrm{zu}$ verstehen. Eine politikwissenschaftliche Disziplin, die diese Gegenstandsbereiche relevant findet, benötigt die Politische Ökonomie.

Die Hinwendung zur polit-ökonomischen Realität von Konflikten um Macht und Wohlstand und ihren globalen Dimensionen wird, so zeichnet sich bereits heute ab, wie von Mastanduno analysiert, durch die Wechselwirkung von gesellschaftlichen Bedingungen und wissenschaftsinternen Dynamiken stattfinden. Abgesehen von der sozialen Frage wird auch die Klimakrise strategische Fragen von Import- und Export und Migrations- und Konfliktbekämpfung mittelfristig immer weiter in den Mittelpunkt stellen. Es ist keine allzu gewagte Prognose, dass Politische Ökonomie und bigger theory bald wieder wichtig sein werden. Das ist, so ist hoffentlich deutlich geworden, eine vielversprechende Zukunft für Konfliktforschung und Internationale Beziehungen.

\section{Literatur}

Außenministerium (2019). Grundprinzipien deutsches Außenpolitik. https://www.auswaertiges-amt.de/de/aussenpolitik/themen/grundprinzipien/216474, Zugegriffen: 04.10.2020.

Babic, M. (2021). Beyond geopolitics? The value of systemic approaches to the 'new' state capitalism. Geopolitics (forthcoming).

Banse, F. (2019). Compact with Africa: Der deutsche Beitrag zur Investitionsliberalisierung und Finanzialisierung in Afrika. PROKLA. Zeitschrift für Kritische Sozialwissenschaft, 49(194), 79-98.

Barrows, L. C. (1976). Faidherbe and Senegal: a critical discussion. African Studies Review, 19 (1), 95-117.

Bernards, N. (2019). 'Latent' surplus populations and colonial histories of drought, groundnuts, and finance in Senegal. Geoforum,

https://doi.org/10.1016/j.geoforum.2019.10.007.

Beyme, K. (1986). Politikwissenschaften in der Bundesrepublik Deutschland.

Entwicklungsprobleme einer Disziplin. Wiesbaden: Springer VS.

Blackwill, R. \& Harris, J. (2016). War by Other Means: Geoeconomics and Statecraft.

Cambridge: Harvard University Press.

Bleek, W. (2001). Geschichte der Politikwissenschaft in Deutschland. München: C.H.Beck Verlag. 
Bonacker, T. (2011). Forschung für oder Forschung über den Frieden? Zum Selbstverständnis der Friedens- und Konfliktforschung, in: Schlotter, Peter/ Wisotzki, Simone (Hrsg.):

Friedens- und Konfliktforschung, Baden-Baden, 46-78.

Braun, B. (2020, June 18). American Asset Manager Capitalism.

https://doi.org/10.31235/osf.io/v6gue

Brühl, T. (2012). Friedensforschung als „Superwissenschaft“ oder Sub-Disziplin? Zum Verhältnis der Friedens- und Konfliktforschung und der Internationalen Beziehungen, in: Zeitschrift für Internationale Beziehungen 19: 1, 171-183.

Bundesregierung. (2020). Rohstoffstrategie der Bundesregierung, https://www.bmwi.de/Redaktion/DE/Downloads/P-R/rohstoffstrategie-derbundesregierung.html, Zugegriffen: 04.10.2020.

Cramer, C., Sender, J. \& Oqubay, A. (2020). African Economic Development: Evidence, Theory, Policy. Oxford: Oxford University Press.

Daase, C. (1996). Vom Ruinieren der Begriffe: Zur Kritik der Kritischen Friedensforschung. in: Meyer, B. 1996. Eine Welt oder Chaos?. Frankfurt: Suhrkamp Verlag.

Daase, C. \& Deitelhoff, N. (2017). Reclaiming the facts?! ZIB-Roundtable auf der IBSektionstagung 2017. https://zib-online.org/2017/09/27/reclaiming-the-facts-zib-roundtableauf-der-ib-sektionstagung-2017/. Zugegriffen: 04.10.2020

Dieter, H. (2018). Deutschlands zweischneidige Außenwirtschaftspolitik. Berlin: Stiftung Wissenschaft und Politik. https://www.swpberlin.org/fileadmin/contents/products/studien/2018S13_dtr.pdf. Zugegriffen: 20.08.2020

Deitelhoff, N. \& Wolf, K. (2009). Der Widerspenstigen Selbst-Zähmung? Zur Professionalisierung der Internationalen Beziehungen in Deutschland. Politische Vierteljahresschrift, 50(3), 451-475.

Drezner, D. (2016). Where have all the big international relations theories gone? Washington Post. 14.09.2016. Zugegriffen: 20.08.2020

Ehrhart, H. \& Staack, M. (2019). (Hrsg.) Sicherheits- und Friedensordnungen in Afrika: Nationale und regionale Herausforderungen. Baden-Baden, Nomos.

Farell, H, \& Newmann, A. (2019). Weaponized Interdependence: How Global Economic Networks Shape State Coercion. International Security, 44 (1), 42-79.

Frankfurter Allgemeine Zeitung (2020) Deutschland fehlt der Wille zu mehr Verantwortung, 4.2.2020, in: https://www.faz.net/aktuell/politik/inland/deutschland-fehlt-der-wille-zu-mehrverantwortung-weltweit-16616957.html.

Göhler, G. \& Zeuner, B. (1991). Kontinuitäten und Brüche in der deutschen Politikwissenschaft. Baden-Baden: Nomos.

Goldthau, A. (2016). Assessing Nord Stream 2: Regulation, geopolitics \& energy security in 
the EU, Central Eastern Europe \& the UK (EUCERS Strategy Paper No. 10). King's College London.

Hansen, P. \& Jonsson, S. (2014). Eurafrica. The Untold History of European Integration and Colonialism. London: Bloomsbury.

Hellmann, G. \& Daase, C. (2014). Helga Haftendorn (geboren 1933). in: Jesse, Eckhard/ Liebold, Sebastian (Hrsg.), Deutsche Politikwissenschaftler - Werk und Wirkung. Von Abendroth bis Zellentin. Baden-Baden: Nomos. 289-301.

Hellmann, G., Wagner, W. \& Baumann, R. (2014). Außenpolitische Diskurse. In: Deutsche Außenpolitik. Grundwissen Politik. Wiesbaden: Springer VS.

Hirsch, J. (1995). Der nationale Wettbewerbsstatt: Staat, Demokratie und Politik im globalen Kapitalismus, Berlin: Edition ID-Archiv.

Jesse, E. \& S. Liebold, S. (2014). Deutsche Politikwissenschaftler - Werk und Wirkung. Von Abendroth bis Zellentin. Baden-Baden: Nomos.

Kastendiek, H. (1977). Die Entwicklung der westdeutschen Politikwissenschaft. Frankfurt: Campus.

Katzenstein, P. (1985). Small States in World Markets: Industrial Policy in Europe. Ithaca: Cornell University Press.

Koddenbrock, K. (2017). Mehr Kapitalismus wagen: Herrschaftsanalyse, jenseits der Anarchie' und die Rolle des Gelds. Politische Vierteljahresschrift, 2, 269-284.

Koddenbrock, K. (2018). Strukturwandel der Globalisierung? Auf der Suche nach Verbindungen zwischen Trump, Brexit und den Strategien Chinas. Zeitschrift für Internationale Beziehungen, 25(2), 126-143.

Koddenbrock, K., Kvangraven, I. \& Sylla, N. (2021). Beyond financialization: The long durée of finance in Senegal and Ghana. Cambridge Journal of Economics, unter Begutachtung.

Lake, D. (2011). Why "Isms" Are Evil. International Studies Quarterly, 2/2011, S. 465-480.

Masala, C. (2016). Weltunordnung: Die globalen Krisen und das Versagen des Westens. München: $\mathrm{CH}$ Beck.

Mastanduno, M. (1998). Economics and Security in Statecraft and Scholarship. International Organization, 52(4), 825-854.

Mello, P. (2019). Von der Bonner zur Berliner Republik: Die „Zivilmacht“ Deutschland im Spiegel parlamentarischer Debatten zu Auslandseinsätzen der Bundeswehr, 1990 bis 2018. In: Klaus Brummer und Friedrich Kießling (Hrsg.) Zivilmacht Bundesrepublik? Bundesdeutsche außenpolitische Rollen vor und nach 1989 aus politik- und geschichtswissenschaftlichen Perspektiven, Baden-Baden: Nomos, 295-316. 
Münchau, W. (2020) There is a void where European Foreign Policy Should be, in: https://www.ft.com/content/edbeed8a-3947-11ea-a6d3-9a26f8c3cba4, 19 Oktober 2020.

Pigeaud, F. \& Sylla, N. (2018). L'arme invisible de la Francafrique: Une Histoire du Franc CFA. Paris: La Découverte.

Rosecrance, R. (1986). The Rise of the Trading State: Commerce and Conquest in the Modern World. New York: Basic Books.

Schieder, S. (2018). Wo sind sie geblieben?. Aus Politik und Zeitgeschichte. Internationale Sicherheit, 36-37.

Schindler, S. (2020). The Task of Critique in Times of Post-Truth Politics, Review of International Studies, 46 (3), 376-394.

Schindler, S. \& Wille, T. (2019). How Can We Criticize International Practices?. International Studies Quarterly, 63(4), 1014-1024.

Schlichte, S. (2012). Die Internationalen Beziehungen als Kirche, die Friedens- und Konfliktforschung als Sekte? ZIB Zeitschrift für Internationale Beziehungen, 19 (1), 143 154.

Schwarzer, D. (2020). Weaponizing the economy. Berlin Policy Journal, 06.01.2020, https://berlinpolicyjournal.com/weaponizing-the-economy/, Zugegriffen: 04.10.2020.

Staack, M. (2000) Handelsstaat Deutschland; Deutsche Außenpolitik in einem neuen internationalen System, Paderborn: Ferdinand Schöningh Verlag.

Sylla, N. \& Koddenbrock, K. (2019). Financialization in the West-African Monetary Union. African Agenda, 22(2), 7-9.

Ten Brink, T. (2008). Geopolitik: Geschichte und Gegenwart kapitalistischer Staatenkonkurrenz. Münster: Westfälisches Dampfboot.

Tooze, A. (2019). Is this the end of the American century? London Review of Books 41 (07), Zugegriffen: 20.08.2020.

Tooze, A. (2020). Whose century? London Review of Books 42 (15), Zugegriffen: 20.08.2020.

Wemheuer-Vogelaar, W. \& Risse, T. (2016). International Relations Scholars in Germany: Young, Internationalised, and Non-Paradigmatic. German Politics, 27 (1), 89-112, DOI: 10.1080/09644008.2016.1253683.

Wiener, A. (2018). Contestation and Constitution of Norms in Global International Relations. Cambridge: Cambridge University Press.

Wendt, A. (1987). The Agent-Structure Problem in International Relations Theory. International Organization, 41 (3). 335-370. 
Ziebura, G. (1984). Weltwirtschaft und Weltpolitik 1922/24 - 1931. Zwischen Rekonstruktion und Zusammenbruch. Frankfurt a. M: Suhrkamp. 